\title{
Slow pulsars from the STScI/NAIC drift scan search
}

\author{
K. M. Xilouris \\ Center Development Lab, NRAO, Charlottesville, VA 22903, USA
}

\author{
A. Fruchter \\ Space Telescope Science Institute, Baltimore, MD 21218, USA \\ D.R. Lorimer, J. Eder, A. Vazquez \\ Arecibo Observatory, HC3 Box 53995, Arecibo, PR 00612, USA
}

\begin{abstract}
The unique sensitivity of the Arecibo telescope at $430 \mathrm{MHz}$ motivated a drift scan search of the visible sky divided up into eight declination strips. Based on an analysis of the data collected in the region assigned to us, eight new long-period pulsars have so far been discovered.
\end{abstract}

\section{The STScI/NAIC drift scan search}

During the recent Gregorian upgrade of the Arecibo telescope, considerable effort was put into drift scan searches of the Arecibo sky $\left(-1^{\circ}<\delta<+39^{\circ}\right)$ for new pulsars. The STScI/NAIC group was assigned declination strips centered at $1.5^{\circ}, 6.5^{\circ}, 11.5^{\circ}, 16.5^{\circ}, 21.5^{\circ}, 26.5^{\circ}, 31.5^{\circ}$, and $36.5^{\circ}$. A list of 20 candidates was compiled from a search in these areas between 1994 and 1998 . We have so far confirmed eight new pulsars as a result of these observations.

The nominal parameters based on the confirmation observations are summarized in Table 1. Barycentric periods have uncertainties of order one unit in the last digit quoted, while a conservative estimate of the uncertainty in the dispersion measures (DM) is $\pm 10 \mathrm{~cm}^{-3} \mathrm{pc}$. The positions are presently uncertain by of order \pm 5 arcmin in right ascension and declination - equivalent to the half power beam size of the telescope at $430 \mathrm{MHz}$.

Although we presently have no long-term estimates of the flux densities of the new pulsars, it is already clear that they are weak sources with typical flux densities of order 0.5 to $1 \mathrm{mJy}$. Some of the initial detections were probably significantly facilitated by flux amplifications due to interstellar scintillation. Inferred $430-\mathrm{MHz}$ luminosities, based on their fluxes and dispersion measures estimates range between 3 and $30 \mathrm{mJy} \mathrm{kpc}^{2}$. These pulsars, along with those discovered by other groups during the Arecibo upgrade, should greatly assist future statistical studies of the low end of the pulsar luminosity function. More accurate measurements of the flux densities, as well as the spin and astrometric parameters for each source are presently underway at Arecibo as part of a regular timing program using the Penn State Pulsar Machine. 


\begin{tabular}{llllll}
\hline PSR & R.A. & $\begin{array}{c}\text { Decl. } \\
(\mathrm{J} 2000)\end{array}$ & $\begin{array}{l}\text { Period } \\
(\mathrm{sec})\end{array}$ & $\begin{array}{l}\text { Epoch } \\
(\mathrm{MJD})\end{array}$ & $\begin{array}{l}\mathrm{DM} \\
\mathrm{cm}^{-3} \mathrm{pc}\end{array}$ \\
\hline $\mathrm{J} 0137+16$ & $01: 37: 31$ & $+16: 55$ & 0.41477 & 51264 & 26 \\
$\mathrm{~J} 0329+16$ & $03: 29: 13$ & $+16: 54$ & 0.8933 & 51257 & 35 \\
& & & & & \\
$\mathrm{~J} 1549+21$ & $15: 49: 41$ & $+21: 14$ & 1.262 & 51335 & 55 \\
$\mathrm{~J} 1822+11$ & $18: 22: 17$ & $+11: 22$ & 1.787 & 51261 & 112 \\
& & & & & \\
$\mathrm{~J} 1838+16$ & $18: 38: 52$ & $+16: 53$ & 1.902 & 51261 & 36 \\
$\mathrm{~J} 1849+06$ & $18: 49: 07$ & $+06: 07$ & 2.219 & 51258 & 236 \\
& & & & & \\
$\mathrm{~J} 1905+06$ & $19: 05: 21$ & $+06: 23$ & 0.9897 & 51261 & 262 \\
$\mathrm{~J} 2040+16$ & $20: 40: 13$ & $+16: 54$ & 0.8656 & 51261 & 51 \\
\hline
\end{tabular}

Table 1. Parameters of the eight newly-discovered pulsars based on the discovery and confirmation observations.
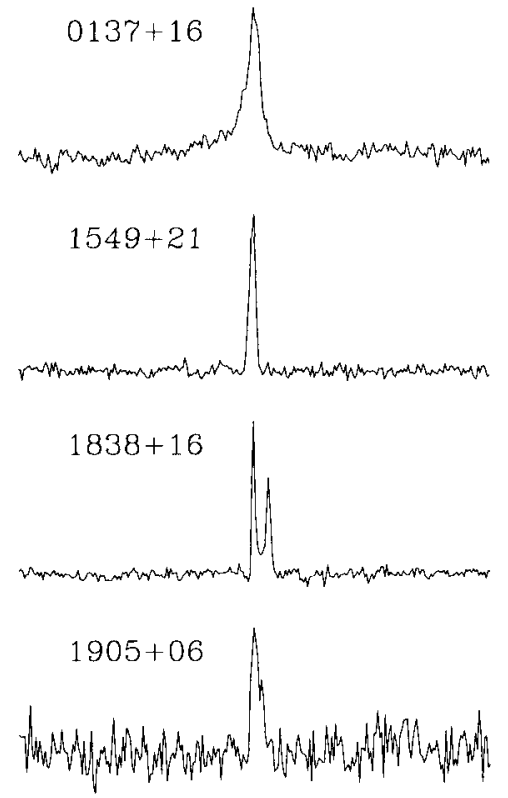
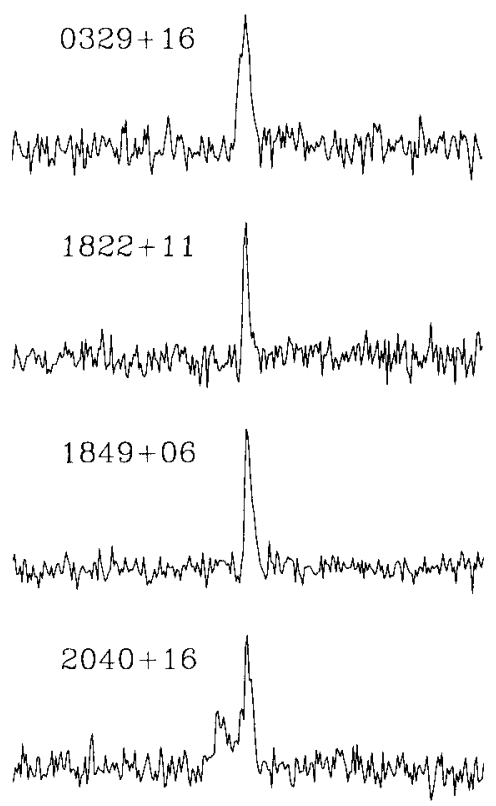

Figure 1. Integrated 430-MHz pulse profiles for the eight, newłydiscovered pulsars. Each profile represents $360^{\circ}$ of rotational phase. 